the joints, characteristic of gouty inflammation (7). Although the results discussed here are essentially based on a mouse model of MSU crystal-induced inflammation, they suggest a general model of the mechanism of gouty inflammation that raises a host of new questions. For instance, it is not clear what cell types respond to IL- $1 \beta$ in the joints. We might anticipate that synoviocytes or endothelial cells play a central role in recruiting neutrophils $(13,14)$, but other cell types may be affected. For example, IL-1 $\beta$ may promote osteoclast activation and differentiation and trigger inflammatory pain hypersensitivity by acting on neurons (15).

Another important issue that remains open is the mechanism controlling the resolution phase in gout. MSU crystalinduced inflammation is generally selflimiting, suggesting that negative feedback loops control the immune response to MSU crystals. Whether such mechanisms involve an inhibition of the maturation of IL- $1 \beta$ or a desensitization to IL- $1 \beta$ responses remains to be investigated. Finally, the study of the physiological function of IL-1 $\beta$ in human gouty inflammation will undoubtedly provide new therapeutic tools to better manage the acute inflam- mation episodes in patients with gout and provide new insights into the pathology of other autoinflammatory diseases linked to deregulated IL- $1 \beta$ production.

\section{Acknowledgments}

We thank Wendy Garrett for discussions and critical reading of the manuscript.

Address correspondence to: Laurie H. Glimcher, 651 Huntington Avenue, Building FXB Room 205, Boston, Massachusetts 02115, USA. Phone: (617) 432-4846; Fax: (617) 4320084; E-mail: lglimche@hsph.harvard.edu.

1. Nuki, G., and Simkin, P.A. 2006. A concise history of gout and hyperuricemia and their treatment. Arthritis Res. Ther. 8(Suppl. 1):S1.

2. Dalbeth, N., and Haskard, D.O. 2005. Mechanisms of inflammation in gout. Rheumatology (Oxford). 44:1090-1096.

3. Faires, J.S., and McCarty, D.J. 1962. Acute arthritis in man and dog after intrasynovial infection of sodium urate crystals. Lancet. 280:682-685.

4. Shi, Y., Evans, J.E., and Rock, K.L. 2003. Molecular identification of a danger signal that alerts the immune system to dying cells. Nature. 425:516-521.

5. Heath, W.R., and Carbone, F.R. 2003. Immunology: dangerous liaisons. Nature. 425:460-461.

6. Rock, K.L., Hearn, A., Chen, C.J., and Shi, Y. 2005. Natural endogenous adjuvants. Springer Semin. Immunopathol. 26:231-246.

7. Chen, C.-J., et al. 2006. MyD88-dependent IL-1 receptor signaling is essential for gouty inflammation stimulated by monosodium urate crystals. J. Clin. Invest. 116:2262-2271. doi:10.1172/JCI28075.

8. Schnare, M., et al. 2001. Toll-like receptors control activation of adaptive immune responses. Nat. Immunol. 2:947-950.

9. O’Neill, L.A. 2006. How Toll-like receptors signal: what we know and what we don't know. Curr. Opin. Immunol. 18:3-9.

10. Martinon, F., and Tschopp, J. 2005. NLRs join TLRs as innate sensors of pathogens. Trends Immunol. 26:447-454.

11. Martinon, F., Petrilli, V., Mayor, A., Tardivel, A., and Tschopp, J. 2006. Gout-associated uric acid crystals activate the NALP3 inflammasome. Nature. 440:237-241.

12. Liu-Bryan, R., Scott, P., Sydlaske, A., Rose, D.M., and Terkeltaub, R. 2005. Innate immunity conferred by Toll-like receptors 2 and 4 and myeloid differentiation factor 88 expression is pivotal to monosodium urate monohydrate crystal-induced inflammation. Arthritis Rheum. 52:2936-2946.

13. Chapman, P.T., et al. 1997. Endothelial activation in monosodium urate monohydrate crystalinduced inflammation: in vitro and in vivo studies on the roles of tumor necrosis factor alpha and interleukin-1. Arthritis Rheum. 40:955-965.

14. Terkeltaub, R., Baird, S., Sears, P., Santiago, R., and Boisvert, W. 1998. The murine homolog of the interleukin-8 receptor CXCR-2 is essential for the occurrence of neutrophilic inflammation in the air pouch model of acute urate crystal-induced gouty synovitis. Arthritis Rheum. 41:900-909.

15. Samad, T.A., et al. 2001. Interleukin-1beta-mediated induction of Cox-2 in the CNS contributes to inflammatory pain hypersensitivity. Nature. 410:471-475

\title{
What's in a name? eNOS and anaphylactic shock
}

\section{Charles J. Lowenstein ${ }^{1}$ and Thomas Michel ${ }^{2}$}

\begin{abstract}
1Department of Medicine, Johns Hopkins University School of Medicine, Baltimore, Maryland, USA. ${ }^{2}$ Cardiovascular Division, Department of Medicine, Brigham and Women's Hospital, Harvard Medical School, Boston, Massachusetts, USA, and Veterans Affairs Boston Healthcare System,
\end{abstract} West Roxbury, Massachusetts, USA.

\begin{abstract}
In this issue of the JCI, a study by Cauwels and colleagues suggests a central role for eNOS, the endothelial isoform of nitric oxide synthase, as a mediator of anaphylaxis (see the related article beginning on page 2244). Why is an enzyme originally described as a physiological mediator of vascular homeostasis implicated in the spectacular vascular collapse that is characteristic of anaphylaxis? And is the eNOS involved in anaphylaxis necessarily exerting its effect solely in the vascular endothelium, or might this "endothelial enzyme" actually be playing a more fundamental role in an entirely different tissue? After all, what's in a name?
\end{abstract}

Mammalian NOS isoforms were originally named after the tissues in which they were first characterized and cloned. Neuronal NOS (nNOS) was used to des-

Nonstandard abbreviations used: nNOS, neuronal NOS; PAF, platelet-activating factor.

Conflict of interest: The authors have declared that no conflict of interest exists.

Citation for this article: J. Clin. Invest. 116:2075-2078 (2006). doi:10.1172/JCI29406. ignate the NOS isoform first purified and cloned from neurons, and eNOS was first isolated and characterized in endothelial cells. The NOS isoform found to be expressed in a variety of immunomodulatory cells after their stimulation with inflammatory cytokines was dubbed the inducible (or inflammation-related) NOS, or iNOS. The genes that encode nNOS, iNOS, and eNOS were officially named after the order of their discovery and characterization: NOS1, NOS2, and NOS3, respectively. However, the appellations of nNOS, iNOS, and eNOS proteins persist in the literature, perhaps for historical reasons, but these terms belie a much broader tissue distribution of these key regulatory proteins (Figure 1). Researchers discovered nNOS in nonneuronal tissues, including skeletal muscle and cardiac muscle (1). The so-called inducible iNOS now appears to be constitutively expressed in some epithelial cells in the lung and in the gastrointestinal tract as well as in myriad other cell types following their immunoactivation (2). Over the years since the original identification of eNOS in endothelial cells $(3,4)$, this "endothelial" isoform has been characterized in diverse cell types, including cardiac 
Blood platelet Inhibition of platelet function

Renal epithelium Regulation of ion transport Local vasoregulation
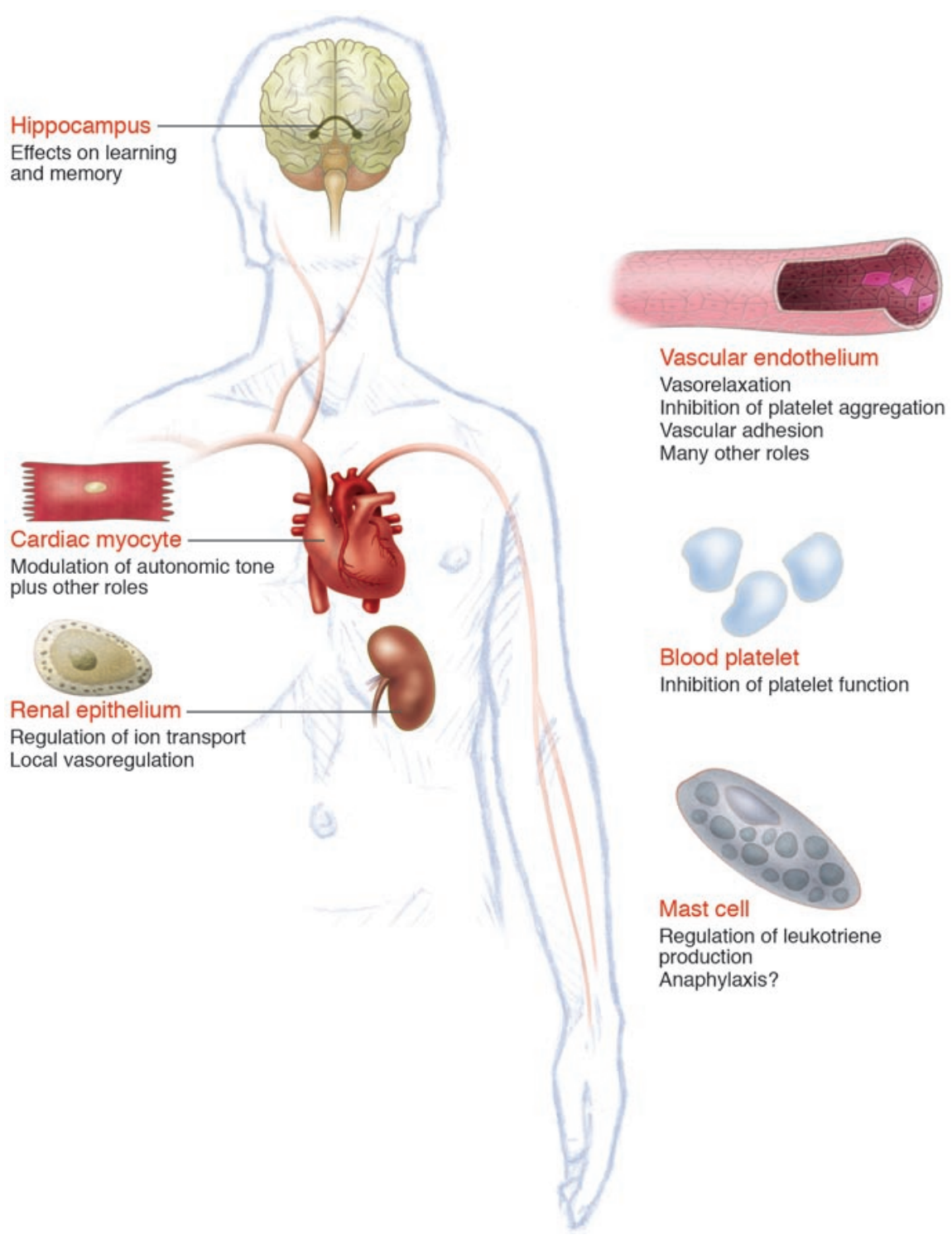

myocytes, blood platelets, hippocampus neurons, renal epithelial cells, and - possibly most relevant for the current topic of anaphylaxis - in mast cells (5).

eNOS has been considered to act as an archetypal signaling enzyme, responding to physiological signals by transiently synthesizing small amounts of NO that reduce blood pressure and inhibit platelet aggregation in response to local homeostatic stimuli (see reviews in refs. 5,6 ). In contrast, the iNOS isoform, with its much greater capacity for NO synthesis in immunoactivated cells, has been viewed as the NOS most likely to respond to pathological stimulation. Since iNOS produces massive quantities of $\mathrm{NO}$ in order to kill pathogens - even to the point where iNOS-generated NO could lead to excessive vascular

\section{Figure 1}

eNOS is expressed not only in the vascular endothelium, but also in blood platelets, cardiac myocytes, hippocampal neurons, kidney, epithelial cells in lung and gut, and mast cells. The diversity of cell types that express eNOS suggests that the eNOS isoform may play multiple physiological and pathophysiological roles (14), regulating vasoconstriction, thrombosis, platelet aggregation, myocyte signaling, and neurotransmission. Recently, eNOS has also been found in mast cells, and findings reported by Cauwels et al. (8) in this issue of the $\mathrm{JCl}$ suggest a role for eNOS in anaphylactic shock.

nition of an antigen; cross-linked IgE binds to Fc receptors on basophils and mast cells, triggering degranulation and release of histamine, proteases, cytokines, and arachidonic acid metabolites. Since cytokines can activate iNOS expression and iNOS produces large amounts of NO, which causes shock in other inflammatory diseases, such as septic shock, it was widely assumed that iNOS mediates anaphylactic shock as well $(10,11)$.

However, Cauwels and colleagues now present evidence suggesting that eNOS, not iNOS, is a critical mediator of anaphylactic shock in mice (8). These investigators studied the pathogenesis of shock by injecting mice with platelet-activating factor (PAF), a phospholipid messenger released by antigen-stimulated mast cells that triggers vascular leakage, hypotension, and death during septic shock and anaphylactic shock (12). The nonselective NOS inhibitor L-NAME blocked the severe hypotension that normally follows PAF administration, suggesting that one of the NOS isoforms plays a critical role in anaphylactic hypotension. NOS-derived $\mathrm{NO}$ activates soluble guanylate cyclase and increases cyclic GMP levels and thereby elicits biological responses in many different tissues. However, the authors note that soluble guanylate cyclase inhibitors do not seem to prevent anaphylaxis and suggest that the effects of this NOS-derived NO are independent of cyclic GMP. To identify the NOS isoform involved, the researchers injected PAF into wild-type, iNOS knockout, and eNOS knockout mice. Virtually all wild-type mice and iNOS knockout mice died after PAF injection, but most eNOS knockout mice survived. These surprising findings suggest that eNOS, not iNOS, mediates anaphylactic shock. What is the underlying mechanism? 


\section{Plausible mechanisms}

One possibility is that anaphylaxis somehow overstimulates eNOS in the endothelium to generate copious amounts of NO, thereby excessively relaxing vascular smooth muscle and leading to hypotension and death. Under physiological conditions, eNOS activity in the vascular endothelium is exquisitely modulated by multiple regulatory pathways that influence the enzyme's posttranslational modifications, subcellular targeting, and protein-protein interactions (reviewed in ref. 14). In normal vascular endothelial cells, low levels of NO are transiently synthesized by eNOS in response to receptor- or flow-mediated increases in intracellular calcium levels, which promote calmodulin binding to eNOS; calmodulin is required for NOS enzyme activation. The activity of eNOS is complexly regulated, with changes in enzyme phosphorylation, $S$-nitrosylation, acylation, and protein-protein interactions each importantly influencing eNOS activity. Of these various posttranslational modifications, phosphorylation of eNOS by protein kinase Akt (also known as PKB) has been particularly well characterized. Kinase Akt is a downstream mediator of the PI3K pathway and directly phosphorylates eNOS at a single residue, leading to significant increases in eNOS enzyme activity (13, 14). In the Cauwels study (8), the authors show that anaphylaxis can be blocked by inhibitors of kinase Akt or of PI3K under conditions in which eNOS phosphorylation is similarly blocked; the authors interpret these data to suggest that kinase Akt may play an important role in anaphylaxis by a direct effect on eNOS phosphorylation. However, it must be noted that both PI3K and kinase Akt have pleiotropic roles in many tissues and few kinase inhibitors have absolute specificity for their target proteins. Moreover, the authors found that mice with targeted deletion of the protein kinase Akt 1 isoform still succumb to anaphylaxis, but note that this may be explained by redundancy with the other Akt isoforms that are still expressed in the Akt $1^{-/-}$mouse. The authors conclude that kinase Akt is necessary for cardiovascular shock in anaphylaxis and suggest that Akt-modulated eNOS phosphorylation in endothelial cells might lead to excessive NO synthesis, leading to vasodilation, shock, and death. This conclusion seems quite plausible, yet other interpretations might still be considered. Indeed, the kinases Akt and PI3K influence diverse intracellular pathways, and it's possible that an anaphylaxis-associated increase in PI3K/Akt-dependent eNOS phosphorylation in endothelial cells may be secondary rather than causal.

We suggest another intriguing possibility, namely that anaphylaxis may involve a novel role for eNOS in nonendothelial tissues, for example, eNOS expressed in mast cells (15). Mast cells are proinflammatory effector cells that initiate and regulate anaphylaxis (16). How might NO derived from eNOS activate mast cell release of anaphylactic mediators? Probably not by controlling vesicle release of histamine: exogenous NO appears to inhibit histamine release from basophils and mast cells (17). Moreover, endogenous NO blocks exocytosis of granules by $S$-nitrosylating key components of the exocytic machinery (18). However, NO can activate the production of inflammatory mediators such as prostaglandins. For example, NO S-nitrosylates COX-2, enhancing production of prostaglandin E2 (19). Thus, NO derived from eNOS might cause hypotension by triggering production of inflammatory mediators rather than by directly relaxing vascular smooth muscle and quite plausibly exerting its manifold downstream effects independent of changes in cyclic GMP. Another mechanism may reflect previously unappreciated roles for eNOS in blood platelets or in cardiac myocytes -2 cell types that express eNOS - that are somehow unmasked by anaphylaxis. However, these conjectures and speculations remain largely unexplored: Cauwels's study provides no information on the results of necropsy following death from anaphylaxis, and many of the experiments look solely at changes in animal survival or blood pressure following anaphylaxis (8). It should be self-evident that death is often a complexly determined outcome.

\section{Implications for anaphylaxis in humans}

What does this study of anaphylaxis in mice tell us about human anaphylaxis? Anaphylaxis in humans and mice share many similarities: both species synthesize IgE, express the FceR1 receptor for IgE on mast cells, and release PAF and other mediators from mast cells. Minor differences in anaphylaxis pathways between the 2 species are unlikely to weaken the significance of the current study (for example, human but not mouse macrophages can express the IgE receptor; ref. 20). The authors employed 2 mod- els of anaphylaxis. The first model relied upon infusion of a single mediator, PAF, a situation unlikely to occur in humans. However, the second model more closely mimics human IgE-mediated anaphylaxis: mice were first sensitized to ovalbumin and then challenged with ovalbumin to induce systemic anaphylaxis. Perhaps NO mediates hypotension following anaphylaxis in humans as well. If so, then agents that inhibit NOS or that scavenge NO might prove useful in treating life-threatening anaphylactic shock. While we must remain mindful of the caveats discussed above, these findings also suggest that inhibitors of PI3K or kinase Akt might plausibly be targets for treatment of anaphylaxis. Finally, this surprising observation that the endothelial isoform of NOS plays an important role in an acute, severe inflammatory disorder should rejuvenate the further study of the roles of eNOS in tissues outside of the vascular endothelium.

Address correspondence to: Thomas Michel, Cardiovascular Division, Brigham and Women's Hospital, Thorn Building, Room 1210A, 75 Francis Street, Boston, Massachusetts 02115, USA. Phone: (617) 7327376; Fax: (617) 732-5132; E-mail: tmichel@ research.bwh.harvard.edu. Or to: Charles J. Lowenstein, 950 Ross Building, 720 Rutland Avenue, Baltimore, Maryland 21205, USA. Phone: (410) 955-1530; Fax: (410) 502-5336; E-mail: clowenst@jhmi.edu.

1. Kobzik, L., Reid, M.B., Bredt, D.S., and Stamler, J.S. 1994. Nitric oxide in skeletal muscle. Nature. 372:546-548.

2. Nathan, C. 1997. Inducible nitric oxide synthase: what difference does it make? J. Clin. Invest. 100:2417-2423.

3. Pollock, J.S., et al. 1991. Purification and characterization of particulate endothelium-derived relaxing factor synthase from cultured and native bovine aortic endothelial cells. Proc. Natl. Acad. Sci. U. S. A. 88:10480-10484.

4. Lamas, S., Marsden, P.A., Li, G.K., Tempst, P., and Michel, T. 1992. Endothelial nitric oxide synthase: molecular cloning and characterization of a distinct constitutive enzyme isoform. Proc. Natl. Acad. Sci. U. S. A. 89:6348-6352.

5. Michel, T., and Feron, O. 1997. Nitric oxide synthases: which, where, how, and why? J. Clin. Invest. 100:2146-2152.

6. Dudzinski, D.M., Igarashi, J., Greif, D., and Michel, T. 2006. The regulation and pharmacology of endothelial nitric oxide synthase. Annu. Rev. Pharmacol. Toxicol. 46:235-276.

7. Fang, F.C. 1997. Perspectives series: host/pathogen interactions. Mechanisms of nitric oxide-related antimicrobial activity. J. Clin. Invest. 99:2818-2825.

8. Cauwels, A., Janssen, B., Buys, E., Sips, P., and Brouckaert, P. 2006. Anaphylactic shock depends on PI3K and eNOS-derived NO. J. Clin. Invest. 116:2244-2251. doi:10.1172/JCI25426.

9. Kemp, S.F., and Lockey, R.F. 2002. Anaphylaxis: a 
review of causes and mechanisms. J. Allergy Clin. Immunol. 110:341-348.

10. Szabo, C., and Thiemermann, C. 1994. Invited opinion: role of nitric oxide in hemorrhagic, traumatic, and anaphylactic shock and thermal injury. Shock. 2:145-155.

11. Szabo, C., et al. 1993. Platelet-activating factor contributes to the induction of nitric oxide synthase by bacterial lipopolysaccharide. Circ. Res. 73:991-999.

12. Ishii, S., et al. 1998. Impaired anaphylactic responses with intact sensitivity to endotoxin in mice lacking a platelet-activating factor receptor. J. Exp. Med. 187:1779-1788.

13. Fulton, D., et al. 1999. Regulation of endothelium- derived nitric oxide production by the protein kinase Akt. Nature. 399:597-601.

14. Dimmeler, S., et al. 1999. Activation of nitric oxide synthase in endothelial cells by Akt-dependent phosphorylation. Nature. 399:601-605.

15. Gilchrist, M., McCauley, S.D., and Befus, A.D. 2004. Expression, localization, and regulation of NOS in human mast cell lines: effects on leukotriene production. Blood. 104:462-469.

16. Galli, S.J., et al. 2005. Mast cells as "tunable" effector and immunoregulatory cells: recent advances. Annu. Rev. Immunol. 23:749-786.

17. Iikura, M., et al. 1998. Exogenous nitric oxide regulates the degranulation of human basophils and rat peritoneal mast cells. Int. Arch. Allergy Immunol. 115:129-136.

18. Matsushita, K., et al. 2003. Nitric oxide regulates exocytosis by $\mathrm{S}$-nitrosylation of $\mathrm{N}$-ethylmaleimidesensitive factor. Cell. 115:139-150.

19. Kim, S.F., Huri, D.A., and Snyder, S.H. 2005. Inducible nitric oxide synthase binds, S-nitrosylates, and activates cyclooxygenase-2. Science. 310:1966-1970.

20. Finkelman, F.D., Rothenberg, M.E., Brandt, E.B., Morris, S.C., and Strait, R.T. 2005. Molecular mechanisms of anaphylaxis: lessons from studies with murine models. J. Allergy Clin. Immunol. 115:449-457; quiz 458.

\title{
HIV and CXCR4 in a kiss of autophagic death
}

\author{
Beth Levine and Donald L. Sodora
}

Departments of Internal Medicine and Microbiology, University of Texas Southwestern Medical Center, Dallas, Texas, USA.

\begin{abstract}
AIDS is characterized by $\mathrm{CD4}^{+} \mathrm{T}$ lymphocyte depletion, yet the mechanisms underlying this central aspect of HIV pathogenesis are still poorly understood. In this issue of the JCI, Espert et al. identify a mechanism by which the HIV envelope glycoprotein can induce death in uninfected $\mathrm{CD}^{+} \mathrm{T}$ cells (see the related article beginning on page 2161). The HIV envelope glycoprotein interacts with CXC chemokine receptor 4 to activate the lysosomal degradation pathway of autophagy, which is necessary for both apoptotic and nonapoptotic cell death.
\end{abstract}

Since the beginning of the AIDS epidemic in the 1980s, even before the HIV virus was identified, physicians and scientists recognized that a cardinal feature of AIDS was the depletion of $\mathrm{CD}^{+} \mathrm{T}$ lymphocytes. Yet nearly a quarter of a century later, our understanding of how $\mathrm{CD}^{+} \mathrm{T}$ cells are depleted in HIV-infected patients remains incomplete. $\mathrm{CD}^{+} \mathrm{T}$ cells are killed by direct HIV infection, but substantial numbers of uninfected $\mathrm{CD} 4^{+} \mathrm{T}$ cells also die in HIV-infected patients. The death of these cells is postulated to result from Fasmediated activation-induced cell death and/or the stimulation of apoptosis in uninfected bystander cells by released or cell surface-expressed HIV gene products including accessory proteins (e.g., Tat, $\mathrm{Vpr}, \mathrm{Vpu}$, and $\mathrm{Nef}$ ) and envelope proteins (reviewed in refs. 1-3).

In this issue of the JCI, Espert et al. describe an advance in understanding how the HIV envelope glycoprotein can

Nonstandard abbreviations used: CCR5, CC chemokine receptor 5; CXCR4, CXC chemokine receptor 4; 3-MA, 3-methyladenine; SDF-1, stromal cell-derived factor 1 .

Conflict of interest: The authors have declared that no conflict of interest exists.

Citation for this article: J. Clin. Invest. 116:2078-2080 (2006). doi:10.1172/JCI29447. kill uninfected $\mathrm{CD}^{+} \mathrm{T}$ lymphocytes (4). By coculturing effector cells that express the HIV envelope glycoprotein with target cells that express CD4 and CXC chemokine receptor 4 (CXCR4), they demonstrate that CXCR4 engagement by the HIV envelope glycoprotein activates a lysosomal degradation pathway known as autophagy. Based on studies with pharmacological and genetic autophagy inhibitors, this activation of autophagy appears necessary both for caspase-dependent, apoptotic death of bystander cells and for caspase-independent, nonapoptotic death, which is presumably directly due to autophagy (Figure 1).

The word autophagy is derived from Greek and means to eat (phagy) oneself (auto). It is an evolutionarily conserved process involving the dynamic rearrangement of subcellular membranes to sequester cytoplasm and organelles for delivery to the lysosome, where the sequestered cargo is degraded and recycled. Autophagy occurs at basal levels in most tissues and contributes to the routine turnover of cytoplasmic components, playing a housekeeping function that is believed to delay aging, protect against neurodegeneration, and potentially function in tumor suppression (5-7). Autophagy is rapidly upregulated in response to different forms of cellular stress. This induction of autophagy may help promote cell survival, either by purging the cell of damaged organelles, toxic metabolites, and intracellular pathogens or by generating the intracellular building blocks required to maintain vital functions during nutrient-limiting conditions (reviewed in ref. 8). However, when very high levels of autophagy are induced, autophagy may also promote cell death through excessive self-digestion and degradation of essential cellular constituents.

There is increasing evidence that complex interrelationships exist between autophagy and the apoptotic cell death pathway (reviewed in ref. 8). Several regulators of apoptosis activation also function as regulators of autophagy activation (e.g., TRAIL, FADD, DAPk, ceramide, class I PI3K/Akt signaling, and Bcl-2 family members). Previously, it has been shown that genetic inhibition of autophagy can activate apoptotic death in nutrient-starved mammalian cells (9), suggesting that autophagy activation can function to prevent apoptosis. Conversely, it has also been suggested that autophagy activation may lead to apoptosis (reviewed in ref. 8); this conclusion was supported by data using a pharmacological inhibitor of autophagy, 3-methyladenine (3-MA), a nucleotide derivative that blocks class III PI3K activity. However, 3-MA can inhibit kinases other than class III PI3K (10), some of which may independently affect death signaling as well as inhibit the mitochondrial permeability transition (11). Now Espert et al. demonstrate that short interfering RNAs specific for 2 different autophagy execution genes (beclin 1 and atg7) can completely 